九州大学学術情報リポジトリ

Kyushu University Institutional Repository

\title{
Studies on the marine yeasts. VI : On some physiological properties of the isolates
}

Suehiro, Sumio

Laboratory of Chemistry of Fishery Product, Faculty of Agriculture, Kyusyu University

Tomi yasu, Yukio

Laboratory of Chemistry of Fishery Product, Faculty of Agriculture, Kyusyu University

https://doi.org/10.5109/22720

出版情報 : 九州大学大学院農学研究院紀要. 13 (1)，pp.213-225，1964-03. Kyushu University バージョン：

権利関係 : 
Journal of the Faculty of Agriculture, Kyushu University, Vol. 13, No. 1

March 30, 1964

Studies on the marine yeasts. VI

On some physiological properties of the isolates

Sumio SUEHIRo* and Yukio TOMIYASU†

The physiological properties of 138 cultures of yeast, isolated from marine muds, ${ }^{3)}$ marine plankton, “? ${ }^{4)}$ and seaweeds, ${ }^{1,5)}$ were investigated : namely, the salt tolerance, the temperature range for the growth, the effect of the addition of $3 \% \mathrm{NaCl}$ on the growth, and the growth in peptone sea water.

\section{The salt tolerance}

The salt tolerance test was made with $3 \%$ Malt Extract (Difco) containing $\mathrm{NaCl}$ ranging in concentration from 5 to $20 \%$. The incubation period ranged from 10 days $(5,10 \%)$ to 20 days $(15,20 \%)$ at $20^{\circ} \mathrm{C}$.

Three cultures were able to grow in the medium containing $20 \%$ $\mathrm{NaCl}$ and eight in $15 \%$. All of them belonged to Torulopsis famata or Candida parapsilosis var. intermedia. It is interesting that all these cultures showing high salt tolerance were isolated from seaweeds only, while the cultures of C.parapsilosis var. intermedia isolated from plankton never showed such high tolerance. All of them, moreover, have maintained the high tolerance after having been cultured on wort agar without $\mathrm{NaCl}$ for ten years. Since the salt concentration of the surface of seaweeds becomes higher in low tide due to evaporation, the yeasts, attaching themselves to the surface, may get such a high degree of tolerance. It is considered, therefore, that the yeasts must always attach themselves to the seaweeds in order to get such high tolerance. Moreover, the other physiological properties of the marine yeasts appear to vary according to the places where they exist.

The temperature range for the growth

The temperature range for the growth was determined by using

* Fishery Research Laboratory, Kyushu University, Tsuyazaki, near Fukuoka.

$\dagger$ Department of Fisheries, Kyushu Univ ersity, Fukuoka. 
$3 \%$ Malt Extract. The incubation period ranged from 2-5 days (above $\left.20^{\circ} \mathrm{C}\right)$ to 20 days $\left(5^{\circ} \mathrm{C}\right)$.

Among 11 cultures of C. tropicalis tested, four, isolated in summer, could grow at $41^{\circ} \mathrm{C}$, while all of them isolated in winter could not grow even at $38^{\circ} \mathrm{C}$, and at $5^{\circ} \mathrm{C}$ most of them isolated in summer were unable to grow or grew poorly. There were no cultures that could grow at $43^{\circ} \mathrm{C}$. From the point of view of the temperature range for the growth, therefore, yeast biota in the sea seems to vary according to seasonal changes.

\section{The effect of the addition of $3 \% \mathrm{NaCl}$}

The effect was tested with Hayduck's media, one containing $3 \%$ $\mathrm{NaCl}$ and the other containing no $\mathrm{NaCl}$, at $20^{\circ} \mathrm{C}$ for five days. The degree of the growth was determined with the turbidity by using photoelectric colorimeter, and the effect was determined by estimating the ratio of the growth in the medium containing $3 \% \mathrm{NaCl}$ to that in the medium containing no $\mathrm{NaCl}$.

Most of the cultures grew better in the medium without $\mathrm{NaCl}$ than in that containing $3 \% \mathrm{NaCl}$. Within the limit of this experiment,

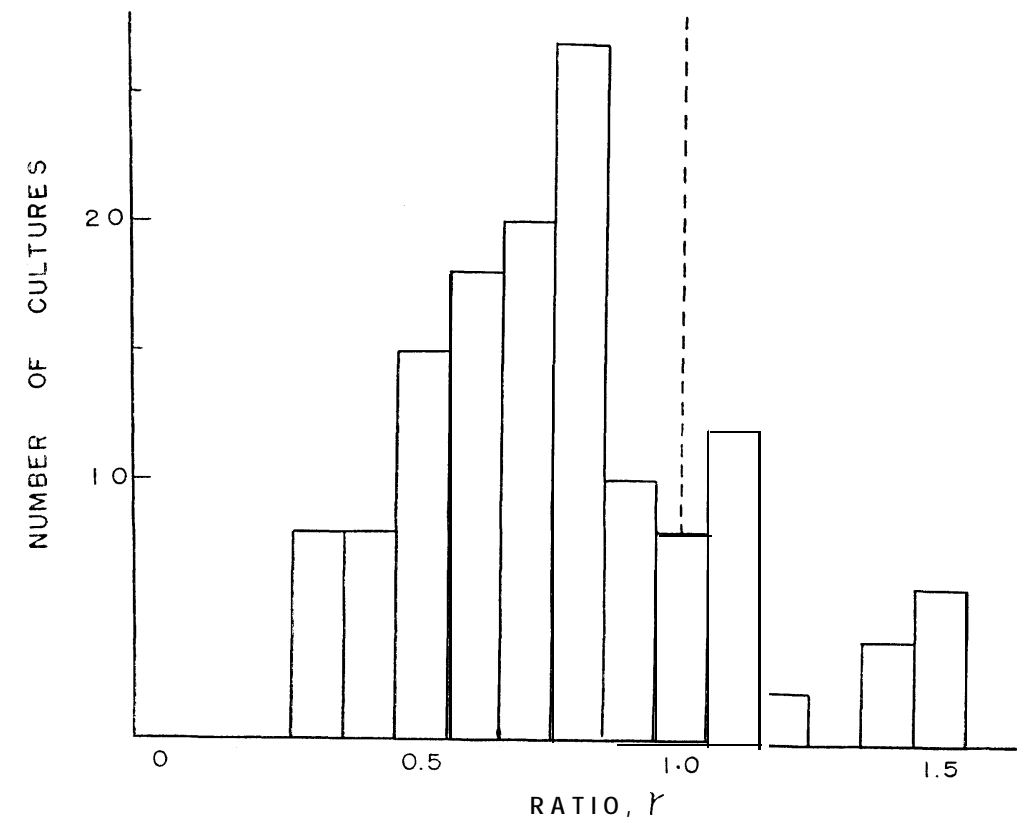

Fig. 1. Frequency distribution of the ratio, $r$, with all the cultures tested.

$*_{r}$ is obtained as the value of the ratio of the growth in the medium containing $3 \% \mathrm{NaCl}$ to that without $\mathrm{NaCl}$. 
it seems that the salt requirement of these cultures are not remarkable. However, the ratio, above mentioned, varied with cultures. This is the reason why this test was carried out. The frequency distribution of the ratio with all the cultures is given in Fig. 1. The maximal number of the cultures was observed at 0.8 (value of the ratio), the distribution itself being definable with genus. The distributions with Candida,Cryptococcus, and Rhodotorula are shown in Fig. 2.

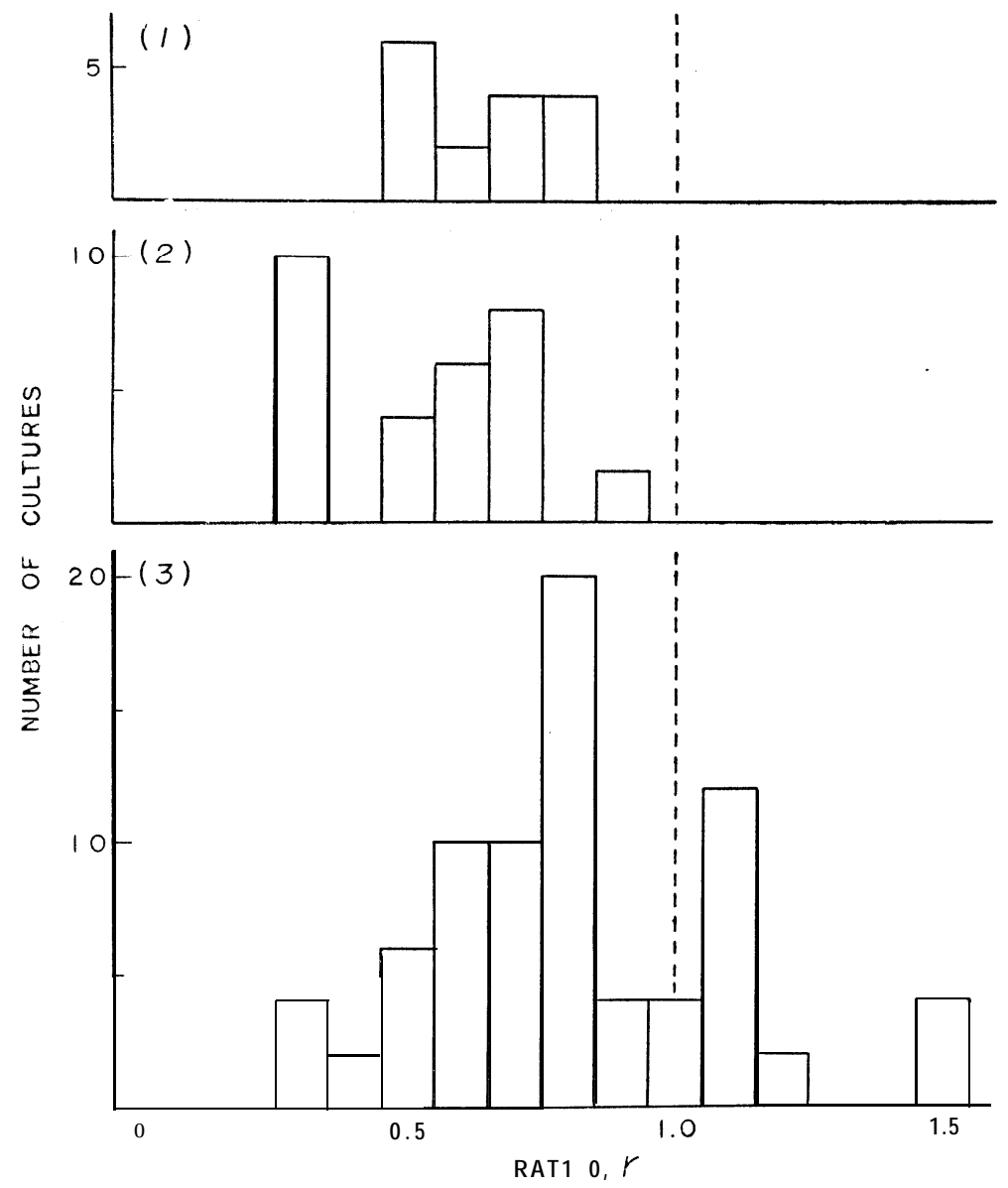

Fig. 2. Frequency distribution of the ratio, $r$, with the cultures of Cryptococcus, Rhodotorula, and Candida.
(1): Cryptococcus,
(2) : Rhodotorula,
(3) : Candida

Some cultures (especially Torulopsis) grew better in the medium containing $3 \% \mathrm{NaCl}$ than in that without $\mathrm{NaCl}$. The cultures of Candida seemed to be more halophylic than those of Cryptococcus and 
Rhodotorula. Though the cultures of Torulopsis were not enough ir. number to be discussed adequately, they seemed to be most halophylic among the cultures tested, and the peak of the distribution appeared to be at 1.0. From the view point of salt requirement, there seemed to be few indigenous marine species among the cultures. It is a wellknown fact that most marine bacteria fail to grow in the medium without $3 \% \mathrm{NaCl}$, but in our studies such cultures of yeast as fail to grow without $\mathrm{NaCl}$ have not been isolated. Even those cultures, showing high salt tolerance as previously mentioned, could grow well in the medium without $\mathrm{NaCl}$. Among the cultures of Candida, some were found to be unable to produce pseudomycelium in the medium without $\mathrm{NaCl}$. On counting the number of the yeasts in marine samples by plating, the ratio of the number of yeast colonies developing in the medium without $\mathrm{NaCl}$ to that developing in the medium containing $3 \% \mathrm{NaCl}$ was always found to be 1: 1 in our experiments. In the case of marine bacteria, however, the ratio was $8: 100{ }^{6)}$ Consequently, the characteristics of indigenous marine species of yeast appear to differ considerably from that of bacteria, and in spite of lacking in strict salt requirement, following cultures may be considered as indigenous marine species :

1) The cultures unable to produce pseudomycelium in the medium not containing $3 \% \mathrm{NaCl}$.

2) The cultures showing high salt tolerance.

3) The cultures growing better in the medium containing $3 \%$ $\mathrm{NaCl}$ than in that without $\mathrm{NaCl}$.

\section{The growth in peptone sea water}

The growth in peptone sea water was determined by using the sea water containing $0.5 \%$ Polypeptone (Wakō Co.) at $20^{\circ} \mathrm{C}$ for five days. The $\mathrm{pH}$ of the medium was adjusted to 7.5 with $\mathrm{NaOH}$.

In spite of poor sugar content in plankton, the marine yeasts utilize it well. These yeasts, therefore, may grow well with amino acids as sole source of carbon. Then, if these cultures are indigenous marine species, they may grow in the medium. As shown in Table 3, a considerable number of the cultures were able to grow well. Most of the cultures of Rhodotorula mucilaginosa grew well, while all the cultures of the other species of the genus were unable to grow or grew poorly. The characteristic of the cultures of the species seems to have some connection with the abundance of the isolates of the species from decaying plankton and seaweeds. 


\section{SUMMARY}

1) Among 138 cultures of yeast tested, three cultures were able to grow in the medium containing $20 \% \mathrm{NaCl}$ and eight in $15 \%$. They belonged to Torulopsis famata or Candida parapsilosis var. intermedia and were isolated from seaweeds only. Such high salt tolerance was never observed on the cultures isolated from plankton. All cultures could grow in the medium containing $5 \% \mathrm{NaCl}$.

2) Most of the cultures grew well at $5-30^{\circ} \mathrm{C}$. Four cultures of Candidatropicalis isolated in summer were able to grow at $41^{\circ} \mathrm{C}$, but all cultures of the species isolated in winter were unable to grow even at $38^{\circ} \mathrm{C}$.

3) Most cultures grew better in the medium not containing $\mathrm{NaCl}$ than in that containing $3 \% \mathrm{NaCl}$. The cultures of Torulopsis and Candida seemed to be more halophylic than those of Cryptococcus and Rhodotorula.

4) A considerable number of the cultures could grow well in peptone sea water ( $\mathrm{pH}$ 7.5). Most cultures of Rhodotorula mucilaginosa grew well in it, but the cultures of the other species of the genus could not grow or grew poorly in it.

\section{REFERENCES}

1) Suehiro, S, 1960. Sci. Bull. Fac. Agr. Kyushu Univ., 17: 443.

2) _...., 1962. Ibid. 20: 101.

3) , 1963. Ibid. 20: 223.

4) —_ and Y. Tomiyasu, 1962. J. Fac. Agr. Kyushu Univ., 12: 163.

5) , and 0. Tanaka, 1962. Ibid. 12: 155.

6) ZoBell, C. E., 1941. J. Marine Research, 4: 42. 
Table 1. The salt tolerance of the isolates.

\begin{tabular}{|c|c|c|c|c|c|c|}
\hline \multirow{2}{*}{ Code } & \multirow{2}{*}{ Taxon } & \multirow{2}{*}{ Source } & \multicolumn{4}{|c|}{$\begin{array}{l}\text { Growth at various con- } \\
\text { centration of } \mathrm{NaCl}\end{array}$} \\
\hline & & & 5 & 10 & 15 & 20 \\
\hline No. 1 & Saccharomyces exiguus & marine mud & H & $\ldots$ & - & - \\
\hline No. 14 & S. cerevisiac & & H & - & - & ... \\
\hline No. 16 & S. delbrueckii & & iti & + & - & - \\
\hline No. 10 & Hansenula anomala & & thi & Ht & - & - \\
\hline No. 24 & Candida parapsilosis & seaweed & tit & + & - & $-\ldots$ \\
\hline No. 164 & & & HH & H & - & - \\
\hline No. 165 & & & mt & H & - & - \\
\hline No. 169 & & & HtH & H & - & - \\
\hline $\begin{array}{l}\text { No. } 170 \\
\text { No. } 177\end{array}$ & & & HH & H & $\underline{-}$ & - \\
\hline No. 181 & & & H & + & $\ldots$ & -- \\
\hline No. 185 & & & m & t & - & - \\
\hline No. 187 & & & itt & + & - & - \\
\hline No. 192 & & & tit & Hit & - & - \\
\hline No. 193 & & & tit & H & - & - \\
\hline No. 194 & & & Ht & Ht & $H$ & + \\
\hline No. 197 & & & H & H & - & - \\
\hline No. 200 & & & Hit & H & - & - \\
\hline No. 203 & & & H & H & H & + \\
\hline 10. 207 & & & & & $\pi T$ & \\
\hline $\begin{array}{ll}\text { No. } & 84 \\
\text { No. } & 98\end{array}$ & & marine plankton & Ht & $\stackrel{t+}{t}$ & - & $\cdots$ \\
\hline No. 100 & & & tit & th & - & - \\
\hline No. 112 & & & +4 & H & - & - \\
\hline No. 114 & & & Hit & $H$ & - & - \\
\hline No. 115 & & & Ht & H & $\ldots$ & - \\
\hline No. 163 & & & H & H & - & - \\
\hline No. 167 & & & tit & tt & - & $\ldots$ \\
\hline $\begin{array}{ll}\text { No. } & 62 \\
\text { No. } & 63\end{array}$ & & T. subtilis* & Hi & + & - & - \\
\hline No. 69 & & & HH & th & -.. & - \\
\hline No. 70 & & & H & $t$ & -.- & - \\
\hline No. 71 & & & H & H & - & - \\
\hline $\begin{array}{l}\text { No. } 31 \\
\text { No. } 106\end{array}$ & C. tropicalis & $\begin{array}{l}\text { marine mud } \\
\text { marine plankton }\end{array}$ & ++ & + & $\cdots$ & - \\
\hline No. 123 & & & tit & + & - & - \\
\hline No. 124 & & & H & + & $\ldots$ & - \\
\hline No. 131 & & & HH & + & - & - \\
\hline No. 132 & & & H & $\overline{11}$ & - & - \\
\hline No & & & tif & $T$ & & \\
\hline No. 139 & & & tit & H & - & - \\
\hline No. 158 & & & HI & H & - & - \\
\hline $\begin{array}{l}\text { No. } 172 \\
\text { No. } 182\end{array}$ & & & $+H$ & $\begin{array}{l}++ \\
+ \\
+\end{array}$ & - & - \\
\hline No. 109 & C. sp. & & tit & H & - & - \\
\hline
\end{tabular}

+ little growth, \# moderate growth,

* Thalassiosira subtilis (marine diatom)2)

Ht heavy growth. 
Table 1. Continued.

\begin{tabular}{|c|c|c|c|c|c|c|}
\hline \multirow{2}{*}{ Code } & \multirow{2}{*}{ Taxon } & \multirow{2}{*}{ Source } & \multicolumn{4}{|c|}{$\begin{array}{l}\text { Growth at various con } \\
\text { centration of Nacl }\end{array}$} \\
\hline & & & 5 & 10 & 15 & 20 \\
\hline $\begin{array}{l}\text { No. } 110 \\
\text { No. } 122 \\
\text { No. } 146 \\
\text { No. } 147 \\
\text { No. } 148\end{array}$ & & & $\begin{array}{l}\text { H } \\
\text { H } \\
\text { H } \\
\text { HH } \\
\text { H }\end{array}$ & $\begin{array}{l}H \\
H \\
H \\
H \\
H\end{array}$ & $\begin{array}{l}- \\
- \\
-\end{array}$ & $\begin{array}{l}- \\
\overline{-} \\
-\end{array}$ \\
\hline $\begin{array}{l}\text { No. } 149 \\
\text { No. } 150 \\
\text { No. } 151 \\
\text { No. } 152 \\
\text { No. } 153\end{array}$ & & & $\begin{array}{l}\text { H } \\
\text { H } \\
\text { H } \\
\text { H } \\
\text { H }\end{array}$ & $\begin{array}{l}H \\
H \\
H \\
H \\
+\end{array}$ & $\begin{array}{l}- \\
- \\
-\end{array}$ & $\begin{array}{l}- \\
- \\
- \\
-\end{array}$ \\
\hline $\begin{array}{lr}\text { No. } & 154 \\
\text { No. } & 159 \\
\text { No. } & 160 \\
\text { No. } & 2 \\
\text { No. } & 99\end{array}$ & C. brumptii & $\begin{array}{l}\text { marine mud } \\
\text { marine plankton }\end{array}$ & $\begin{array}{l}\text { H } \\
\text { H } \\
\text { HH } \\
\text { H } \\
\text { H }\end{array}$ & $\begin{array}{l}H \\
H \\
H \\
H \\
+ \\
H\end{array}$ & $\begin{array}{l}- \\
- \\
-\end{array}$ & $\begin{array}{l}\overline{-} \\
\overline{-} \\
-\end{array}$ \\
\hline $\begin{array}{lr}\text { No. } & 117 \\
\text { No. } & 82 \\
\text { No. } & 17 \\
\text { No. } & 29 \\
\text { No. } & 34\end{array}$ & $\begin{array}{l}\text { C. solani } \\
\text { C. mycoderma } \\
\text { C. albicans } \\
\text { C. guilliermondii }\end{array}$ & $\begin{array}{l}\text { marine plankton } \\
\text { marine mud } \\
\text { seaweed } \\
\text { marine mud }\end{array}$ & $\begin{array}{l}\text { HH } \\
\text { H } \\
\text { H } \\
\text { H } \\
\text { H }\end{array}$ & $\begin{array}{l}H \\
H \\
H\end{array}$ & $\begin{array}{l}- \\
\cdots \\
- \\
-\end{array}$ & $\begin{array}{l}- \\
- \\
- \\
-\end{array}$ \\
\hline $\begin{array}{lr}\text { No. } & 7 \\
\text { No. } & 66 \\
\text { No. } & 67 \\
\text { No. } & 68 \\
\text { No. } & 36\end{array}$ & $\begin{array}{l}\text { C. intermedia } \\
\text { C. lipolytica } \\
\text { Rhodotonula mucilaginosa }\end{array}$ & $\begin{array}{l}\text { marine mud } \\
\text { T. subtilis } \\
\text { marine mud }\end{array}$ & $\begin{array}{l}\text { H } \\
+H \\
H \\
H \\
H\end{array}$ & $\begin{array}{l}+ \\
H \\
H \\
H \\
H\end{array}$ & $\begin{array}{l}- \\
- \\
-\end{array}$ & $\begin{array}{l}- \\
- \\
-\end{array}$ \\
\hline $\begin{array}{ll}\text { No. } & 3 z \\
\text { No. } & 61 \\
\text { No. } & 78 \\
\text { No. } & 80 \\
\text { No. } & 89\end{array}$ & & $\begin{array}{l}T . \text { subtilis } \\
\text { marine plankton }\end{array}$ & $\begin{array}{l}\text { H } \\
\text { H } \\
\text { H } \\
\text { H } \\
\text { H }\end{array}$ & $\begin{array}{l}- \\
- \\
-\end{array}$ & $\begin{array}{l}- \\
- \\
-\end{array}$ & $\begin{array}{l}- \\
- \\
- \\
-\end{array}$ \\
\hline $\begin{array}{l}\text { No. } 127 \\
\text { No. } 128 \\
\text { No. } 136 \\
\text { No. } 161 \\
\text { No. } 171\end{array}$ & & seaweed & $\begin{array}{l}\text { H } \\
\text { H } \\
\text { H } \\
\text { H }\end{array}$ & $\begin{array}{l}- \\
\bar{H} \\
+ \\
+\end{array}$ & $\begin{array}{l}- \\
- \\
-\end{array}$ & $\begin{array}{l}- \\
- \\
-\end{array}$ \\
\hline $\begin{array}{l}\text { No. } 174 \\
\text { No. } 179 \\
\text { No. } 188 \\
\text { No. } 190 \\
\text { No. } 195\end{array}$ & & & $\begin{array}{l}\text { H } \\
\text { H } \\
\text { H } \\
\text { H } \\
\text { H }\end{array}$ & $\begin{array}{l}- \\
\overline{-} \\
+\end{array}$ & $\begin{array}{l}- \\
- \\
-\end{array}$ & $\begin{array}{l}- \\
\overline{-} \\
-\end{array}$ \\
\hline $\begin{array}{l}\text { No. } 201 \\
\text { No. } 104 \\
\text { No. } 88 \\
\text { No. } 94 \\
\text { No. } 95\end{array}$ & $\begin{array}{l}\text { Rh. minuta } \\
\text { Rh. flava }\end{array}$ & $\begin{array}{l}\text { marine plankton } \\
\text { marine plankton }\end{array}$ & $\begin{array}{l}\text { H } \\
\text { H } \\
\text { H } \\
\text { H } \\
\text { H }\end{array}$ & $\begin{array}{l}- \\
- \\
-\end{array}$ & $\begin{array}{l}- \\
- \\
-\end{array}$ & $\begin{array}{l}- \\
-- \\
- \\
-\end{array}$ \\
\hline $\begin{array}{l}\text { No. } 77 \\
\text { No. } 85\end{array}$ & Rh. glutinis & marine plankton & H & - & - & - \\
\hline
\end{tabular}


Table 1. Continued.

\begin{tabular}{|c|c|c|c|c|c|c|}
\hline \multirow{2}{*}{ Code } & \multirow{2}{*}{ Taxon } & \multirow{2}{*}{ Source } & \multicolumn{4}{|c|}{$\begin{array}{l}\text { Growth at various con- } \\
\text { centration of } \mathrm{NaCl}\end{array}$} \\
\hline & & & 5 & 10 & 15 & 20 \\
\hline $\begin{array}{lr}\text { No. } & 162 \\
\text { No. } & 35 \\
\text { No. } & 64 \\
\text { No. } & 65 \\
\text { No. } & 72\end{array}$ & Cryptococcus laurentii & $\begin{array}{l}\text { marine mud } \\
\text { T. subtilis }\end{array}$ & $\begin{array}{l}\text { H } \\
\text { H } \\
H \\
H \\
H\end{array}$ & $\begin{array}{l}+t \\
+ \\
+ \\
+\end{array}$ & $\begin{array}{l}- \\
- \\
- \\
-\end{array}$ & $\begin{array}{l}- \\
I \\
-\end{array}$ \\
\hline $\begin{array}{lr}\text { No. } & 79 \\
\text { No. } & 83 \\
\text { No. } & 92 \\
\text { No. } 111 \\
\text { No. } 143\end{array}$ & & marine plankton & $\begin{array}{l}\text { HH } \\
\text { H } \\
\text { H } \\
\text { HH } \\
\text { HH }\end{array}$ & $\frac{H}{H}$ & $\begin{array}{l}- \\
- \\
-\end{array}$ & $\begin{array}{l}- \\
- \\
-\end{array}$ \\
\hline $\begin{array}{l}\text { No. } 176 \\
\text { No. } 178 \\
\text { No. } 108 \\
\text { No. } 119 \\
\text { No. } 120\end{array}$ & Cr. albidus & $\begin{array}{l}\text { seaweed } \\
\text { marine plankton }\end{array}$ & $\begin{array}{l}\text { HH } \\
\text { HH } \\
\text { HH } \\
\text { HH } \\
\text { HH }\end{array}$ & $\begin{array}{l}\overline{+} \\
- \\
-\end{array}$ & $\begin{array}{l}- \\
\overline{-} \\
\overline{-}\end{array}$ & $\begin{array}{l}- \\
- \\
- \\
-\end{array}$ \\
\hline $\begin{array}{l}\text { No. } 125 \\
\text { No. } 133 \\
\text { No. } 183 \\
\text { No. } 85 \\
\text { No. } 17 \overline{5}\end{array}$ & Cr. diffluens & $\begin{array}{l}\text { marine plankton } \\
\text { seaweed } \\
\text { marine plankton } \\
\text { seaweed }\end{array}$ & $\begin{array}{l}\text { H } \\
\text { Ht } \\
\text { HH } \\
\text { H } \\
\text { HH }\end{array}$ & $\begin{array}{l}- \\
- \\
- \\
-\end{array}$ & $\begin{array}{l}- \\
- \\
-\end{array}$ & $\begin{array}{l}- \\
- \\
- \\
-\end{array}$ \\
\hline $\begin{array}{lr}\text { No. } & 93 \\
\text { No. } 191 \\
\text { No. } 101 \\
\text { No. } 156 \\
\text { No. } \quad 5\end{array}$ & $\begin{array}{l}\mathrm{Cr} \text {. neoformans } \\
\mathrm{Cr} \text {. luteolus } \\
\text { Torulopsis famata }\end{array}$ & $\begin{array}{l}\text { marine plankton } \\
\text { seaweed } \\
\text { marine plankton } \\
\text { seaweed }\end{array}$ & $\begin{array}{l}\text { H } \\
\text { H } \\
\text { H } \\
\text { H } \\
\text { HH }\end{array}$ & $\frac{\bar{t}}{\bar{t}}$ & $\begin{array}{l}- \\
\overline{-} \\
\bar{H}\end{array}$ & $\begin{array}{l}- \\
- \\
- \\
-\end{array}$ \\
\hline $\begin{array}{l}\text { No. } 168 \\
\text { No. } 180 \\
\text { No. } 186 \\
\text { No. } 184 \\
\text { No. } 196\end{array}$ & & & $\begin{array}{l}\text { HH } \\
\text { H } \\
\text { H } \\
\text { H } \\
\text { H }\end{array}$ & $\begin{array}{l}\text { H } \\
\text { H } \\
\text { H } \\
\text { H } \\
H\end{array}$ & $\begin{array}{l}\text { H } \\
\text { Ht } \\
\text { Ht } \\
-\end{array}$ & $\frac{-}{E}$ \\
\hline $\begin{array}{l}\text { No. } 199 \\
\text { No. } 202 \\
\text { No. } 74 \\
\text { No. } 75 \\
\text { No. } 76\end{array}$ & T. inconspicua & T. subtilis & $\begin{array}{l}\text { H } \\
\text { H } \\
\text { HH } \\
\text { HH } \\
\text { HH }\end{array}$ & $\underline{\text { HH }}$ & $\begin{array}{l}- \\
\text { HI } \\
-\end{array}$ & $\begin{array}{l}- \\
- \\
- \\
-\end{array}$ \\
\hline $\begin{array}{l}\text { No. } 116 \\
\text { No. } 20 \\
\text { No. } 27 \\
\text { No. } 129 \\
\text { No. } 130\end{array}$ & $\begin{array}{l}\text { Trichosporon behrendii } \\
\text { Tr. cutaneum }\end{array}$ & $\begin{array}{l}\text { marine plankton } \\
\text { seaweed } \\
\text { seaweed } \\
\text { marine plankton }\end{array}$ & $\begin{array}{l}\text { H } \\
\text { H } \\
H \\
H \\
H\end{array}$ & $\begin{array}{l}+ \\
+ \\
+ \\
-\end{array}$ & $\begin{array}{l}- \\
- \\
- \\
- \\
-\end{array}$ & $\begin{array}{l}- \\
- \\
- \\
-\end{array}$ \\
\hline $\begin{array}{lr}\text { No. } & 189 \\
\text { No. } & 18 \\
\text { No. } 173 \\
\text { No. } 22 \\
\text { No. } 155\end{array}$ & $\begin{array}{l}\text { Tr. infestans } \\
\text { Tr. pullulans } \\
\text { Black yeast }\end{array}$ & $\begin{array}{l}\text { seaweed } \\
\text { seaweed } \\
\text { marine mud } \\
\text { marine plankton }\end{array}$ & $\begin{array}{l}\text { H } \\
\text { HH } \\
\text { HH } \\
\text { H } \\
\text { H }\end{array}$ & $\begin{array}{l}+ \\
+ \\
+ \\
+ \\
+\end{array}$ & $\begin{array}{l}- \\
- \\
- \\
-\end{array}$ & $\begin{array}{l}- \\
- \\
- \\
-\end{array}$ \\
\hline No. 144 & yeast-like fungi & marine plankton & H & + & - & - \\
\hline
\end{tabular}


Table 2. The temperature range for the growth.

\begin{tabular}{|c|c|c|c|c|c|c|c|}
\hline \multirow{2}{*}{ Code } & \multirow{2}{*}{ Taxon } & \multicolumn{6}{|c|}{ Growth at various temperature } \\
\hline & & 5 & 25 & 30 & 35 & 38 & 41 \\
\hline No. 1 & \multirow{41}{*}{$\begin{array}{l}\text { Saccharomyces exiguus } \\
\text { S. cerevisiae } \\
\text { S. delbrueckii } \\
\text { Hansenula anomala } \\
\text { Candida parapsilosis } \\
\quad \text { var. intermedial }\end{array}$} & H & H & H & - & - & - \\
\hline $\begin{array}{ll}\text { No. } & 14\end{array}$ & & H & H & Ht & - & - & - \\
\hline No. 16 & & H & HI & Ht & - & - & - \\
\hline No. 10 & & it & Hit & H & - & - & - \\
\hline No. 24 & & H & HH & H & - & - & - \\
\hline No. 164 & & Ht & H & H & - & - & - \\
\hline No. 165 & & Ht & H & H & - & - & - \\
\hline No. 169 & & Ht & 卅 & H & $\overline{-}$ & - & - \\
\hline No. 170 & & H & $\begin{array}{l}\text { Hit } \\
\text { tht }\end{array}$ & H & H & - & - \\
\hline No. 176 & & \#t & & & & - & \\
\hline No. 181 & & H & H & H & $\overline{-11}$ & 一 & - \\
\hline No. 185 & & H & H & H & HI & - & - \\
\hline No. 187 & & H & H & H & H & - & - \\
\hline $\begin{array}{l}\text { No. } 192 \\
\text { No. } 193\end{array}$ & & 册 & 开 & H & - & - & - \\
\hline & & & & & & & \\
\hline No. 194 & & H & Ht & III & - & 二 & - \\
\hline No. 197 & & HIt & m & $\bar{H}$ & E & - & - \\
\hline $\begin{array}{l}\text { No. } 200 \\
\text { No. } 203\end{array}$ & & 开 & 开 & H & - & - & $\ldots$ \\
\hline $\begin{array}{l}\text { No. } 203 \\
\text { No. } 204\end{array}$ & & H & HH & $\mathrm{Ht}$ & H & H & - \\
\hline No. 84 & & H & HI & H & - & - & - \\
\hline No. 98 & & H & HH & \# & + & - & - \\
\hline No. 100 & & $\overline{-}$ & H & m & \# & $\bar{H}$ & - \\
\hline $\begin{array}{l}\text { No. } 112 \\
\text { No. } 114\end{array}$ & & 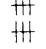 & m & 世 & 世 & H & - \\
\hline No 115 & & \# & $H$ & H & $H$ & - & - \\
\hline $\begin{array}{ll}N & 110 . \\
\text { no. }\end{array}$ & & HH & $H$ & $\mathrm{HH}$ & H & - & - \\
\hline No. 167 & & HI & \# & $\mathrm{HH}$ & H & - & - \\
\hline No. 62 & & H & m & H & - & - & - \\
\hline No. 63 & & H & m & H & & & \\
\hline No. 69 & & H & H & H & - & - & - \\
\hline No. 70 & & H & H & m & - & - & - \\
\hline No. 71 & & H & HI & \# & $\overline{4}$ & - & - \\
\hline 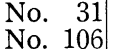 & & + & mit & $\begin{array}{l}\text { m } \\
\text { H }\end{array}$ & H & - & - \\
\hline & & & & & & & \\
\hline $\begin{array}{l}\text { No. } 123 \\
\text { No. } 124\end{array}$ & & + & 开 & 册 & m & 册 & \#\# \\
\hline No. 131 & & + & $\mathrm{Ht}$ & H & H & m & - \\
\hline No. 132 & & H & H & 世 & + & - & $\overline{-}$ \\
\hline No. 135 & & + & m & $\mathrm{mt}$ & m & m & mit \\
\hline No. 139 & & HH & Hit & $\mathrm{HH}$ & HH & + & - \\
\hline No. 158 & & $t$ & \# & H & H & $\mathrm{H}$ & H \\
\hline No. 172 & & H & m & Ht & \# & - & E \\
\hline No. 182 & & At & m & II & II & - & - \\
\hline No. 109 & \multirow{3}{*}{ C. sp. } & H & Ht & m & & & \\
\hline No. 110 & & HI & $\mathrm{Ht}$ & H & - & - & - \\
\hline No. 122 & & $\mathrm{HH}$ & Ht & + & - & - & - \\
\hline
\end{tabular}


Table 2. Continued.

\begin{tabular}{|c|c|c|c|c|c|c|c|}
\hline \multirow{2}{*}{ Code } & \multirow{2}{*}{ Taxon } & \multicolumn{6}{|c|}{ Growth at various temperature } \\
\hline & & 5 & 25 & 30 & 35 & 38 & 41 \\
\hline $\begin{array}{l}\text { No. } 146 \\
\text { No. } 147 \\
\text { No. } 148 \\
\text { No. } 149 \\
\text { No. } 150\end{array}$ & & $\begin{array}{l}\text { H } \\
H \\
H \\
H \\
H\end{array}$ & $\begin{array}{l}\text { Ht } \\
\text { Ht } \\
+H \\
\text { Ht } \\
\text { Ht }\end{array}$ & $\frac{H}{H}$ & $\frac{-}{+}$ & $\begin{array}{l}- \\
- \\
-\end{array}$ & $\begin{array}{l}- \\
- \\
-\end{array}$ \\
\hline $\begin{array}{l}\text { No. } 151 \\
\text { No. } 152 \\
\text { No. } 153 \\
\text { No. } 154 \\
\text { No. } 159\end{array}$ & & $\begin{array}{l}H+ \\
H \\
H \\
H \\
H\end{array}$ & $\begin{array}{l}\text { Ht } \\
\text { HH } \\
\text { Ht } \\
\text { HH } \\
\text { Ht }\end{array}$ & $\begin{array}{l}\text { H } \\
+ \\
+1 \\
+ \\
\text { Ht }\end{array}$ & $\frac{-}{+1}$ & $\begin{array}{l}= \\
- \\
-\end{array}$ & $\begin{array}{l}- \\
- \\
-\end{array}$ \\
\hline $\begin{array}{lr}\text { No. } & 160 \\
\text { No. } & 99 \\
\text { No. } & 117 \\
\text { No. } & 2 \\
\text { No. } & 82\end{array}$ & $\begin{array}{l}\text { C. brumptii } \\
\text { C. solani }\end{array}$ & $\begin{array}{l}+1+ \\
+ \\
+ \\
+ \\
+\end{array}$ & $\begin{array}{l}\text { Ht } \\
\text { HH } \\
\text { Ht } \\
\text { H } \\
\text { H }\end{array}$ & $\begin{array}{l}\text { Ht } \\
\text { H } \\
\text { Ht } \\
\text { Ht } \\
\text { Ht }\end{array}$ & $\frac{H}{-}$ & $\begin{array}{l}H \\
- \\
- \\
-\end{array}$ & $\begin{array}{l}- \\
- \\
-\end{array}$ \\
\hline $\begin{array}{lr}\text { No. } & 17 \\
\text { No. } & 29 \\
\text { No. } & 34 \\
\text { No. } & 7 \\
\text { No. } & 66\end{array}$ & $\begin{array}{l}\text { C. mycoderma } \\
\text { C. albicans } \\
\text { C. guilliermondii } \\
\text { C. intermedia } \\
\text { C. lipolytica }\end{array}$ & $\begin{array}{l}H \\
\bar{H} \\
+\end{array}$ & $\begin{array}{l}\text { H } \\
H \\
H \\
H \\
H\end{array}$ & $\begin{array}{l}H \\
H \\
H \\
H \\
H \\
H\end{array}$ & $\begin{array}{l}- \\
\text { HH } \\
\text { HH } \\
\text { HH }\end{array}$ & $\begin{array}{l}\text { - } \\
\text { Hit } \\
-\end{array}$ & E \\
\hline $\begin{array}{ll}\text { No. } & 67 \\
\text { No. } & 68 \\
\text { No. } & 36 \\
\text { No. } & 38 \\
\text { No. } & 61\end{array}$ & Rhodotorula mucilaginosa & $\begin{array}{l}H \\
H \\
H \\
H \\
H\end{array}$ & $\begin{array}{l}\text { Hit } \\
\text { Ht } \\
H \\
H+ \\
H\end{array}$ & $\begin{array}{l}\text { HH } \\
\frac{H+}{+} \\
+\end{array}$ & $\begin{array}{l}- \\
- \\
- \\
-\end{array}$ & $\begin{array}{l}- \\
- \\
-\end{array}$ & $\begin{array}{l}- \\
- \\
-\end{array}$ \\
\hline $\begin{array}{lr}\text { No. } & 78 \\
\text { No. } & 80 \\
\text { No. } & 89 \\
\text { No. } & 127 \\
\text { No. } & 128\end{array}$ & & $\begin{array}{l}H+ \\
H \\
H \\
H \\
H\end{array}$ & $\begin{array}{l}\text { Ht } \\
+H \\
H+H \\
H \\
H\end{array}$ & $\begin{array}{l}- \\
\bar{t} \\
\text { Hit }\end{array}$ & $\begin{array}{l}- \\
- \\
-\end{array}$ & $\begin{array}{l}- \\
- \\
-\end{array}$ & - \\
\hline $\begin{array}{l}\text { No. } 136 \\
\text { No. } 161 \\
\text { No. } 171 \\
\text { No. } 174 \\
\text { No. } 179\end{array}$ & & $\begin{array}{l}\text { Hit } \\
\text { Hit } \\
\text { Hit } \\
+1+ \\
\text { Hit }\end{array}$ & $\begin{array}{l}H+ \\
H \\
H \\
H \\
H \\
H\end{array}$ & $\frac{H}{+}$ & $\begin{array}{l}- \\
- \\
-\end{array}$ & - & - \\
\hline $\begin{array}{l}\text { No. } 188 \\
\text { No. } 190 \\
\text { No. } 195 \\
\text { No. } 201 \\
\text { No. } 104\end{array}$ & Rh. minuta & $\begin{array}{l}H \\
H \\
H \\
H \\
+\end{array}$ & $\begin{array}{l}\text { Hi } \\
H \\
H \\
H \\
H\end{array}$ & $\begin{array}{l}- \\
+ \\
+ \\
+ \\
+\end{array}$ & $\begin{array}{l}- \\
- \\
-\end{array}$ & - & - \\
\hline $\begin{array}{ll}\text { No. } & 88 \\
\text { No. } & 94 \\
\text { No. } & 95 \\
\text { No. } & 77 \\
\text { No. } & 85\end{array}$ & $\begin{array}{l}\text { Rh. flava } \\
\text { Rh. glutinis }\end{array}$ & $\begin{array}{l}\text { Ht } \\
H \\
H \\
H \\
H\end{array}$ & $\begin{array}{l}H+H \\
H \\
H \\
H \\
H\end{array}$ & $\begin{array}{l}H \\
H \\
H \\
H \\
H\end{array}$ & $\begin{array}{l}- \\
- \\
-\end{array}$ & $\begin{array}{l}- \\
- \\
-\end{array}$ & E \\
\hline $\begin{array}{l}\text { No. } 162 \\
\text { No. } \\
35\end{array}$ & Cryptococcus laurentii & $\begin{array}{l}\text { Ht } \\
\text { Ht }\end{array}$ & Ht & - & - & - & - \\
\hline
\end{tabular}


Table 2. Continued.

\begin{tabular}{|c|c|c|c|c|c|c|c|}
\hline \multirow{2}{*}{ Code } & \multirow{2}{*}{ Taxon } & \multicolumn{6}{|c|}{ Growth at various temperature } \\
\hline & & 5 & 25 & 30 & 35 & 38 & 41 \\
\hline $\begin{array}{ll}\text { No. } & 64 \\
\text { No. } & 65 \\
\text { No. } & 72 \\
\text { No. } & 79 \\
\text { No. } & 83\end{array}$ & & $\begin{array}{l}\text { H } \\
\text { H } \\
\text { Ht } \\
\text { Ht } \\
\text { H }\end{array}$ & $\begin{array}{l}H \\
H \\
H \\
H \\
H \\
H\end{array}$ & $\begin{array}{l}- \\
- \\
-\end{array}$ & $\begin{array}{l}- \\
- \\
-\end{array}$ & $\begin{array}{l}- \\
- \\
- \\
-\end{array}$ & $\begin{array}{l}- \\
- \\
- \\
-\end{array}$ \\
\hline $\begin{array}{l}\text { No. } 92 \\
\text { No. } 111 \\
\text { No. } 143 \\
\text { No. } 176 \\
\text { No. } 178\end{array}$ & & $\frac{\mathrm{HH}}{\mathrm{HH}}$ & $\begin{array}{l}\text { H } \\
\text { H } \\
H \\
H H \\
H\end{array}$ & $\begin{array}{l}\overline{-} \\
\bar{H} \\
-\end{array}$ & $\begin{array}{l}- \\
\overline{-} \\
-\end{array}$ & $\begin{array}{l}\overline{-} \\
\bar{z} \\
\overline{-}\end{array}$ & $\begin{array}{l}- \\
- \\
-\end{array}$ \\
\hline $\begin{array}{l}\text { No. } 108 \\
\text { No. } 119 \\
\text { No. } 120 \\
\text { No. } 125 \\
\text { No. } 133\end{array}$ & Cr. albidus & $\begin{array}{l}H \\
H \\
H \\
H \\
H\end{array}$ & $\begin{array}{l}\text { H } \\
\text { H } \\
H \\
H \\
H\end{array}$ & $\begin{array}{l}H \\
H \\
+ \\
+ \\
+\end{array}$ & $\begin{array}{l}- \\
- \\
-\end{array}$ & $\begin{array}{l}\overline{-} \\
\overline{-}\end{array}$ & $\begin{array}{l}- \\
\overline{-} \\
-\end{array}$ \\
\hline $\begin{array}{lr}\text { No. } & 183 \\
\text { No. } & 86 \\
\text { No. } & 175 \\
\text { No. } & 93 \\
\text { No. } & 191\end{array}$ & $\begin{array}{l}\text { Cr. diffluens } \\
\text { Cr. neoformans }\end{array}$ & $\begin{array}{l}H \\
H \\
H \\
H \\
H\end{array}$ & $\begin{array}{l}\text { H } \\
H \\
H \\
H \\
H \\
H\end{array}$ & $\begin{array}{l}H \\
H \\
H \\
H \\
H\end{array}$ & $\begin{array}{l}- \\
\overline{-} \\
=\end{array}$ & $\begin{array}{l}- \\
- \\
- \\
-\end{array}$ & $\begin{array}{l}- \\
\overline{-} \\
-\end{array}$ \\
\hline $\begin{array}{lr}\text { No. } 101 \\
\text { No. } 156 \\
\text { No. } & 5 \\
\text { No. } 168 \\
\text { No. } 180\end{array}$ & $\begin{array}{l}\text { Cr. luteolus } \\
\text { Torulopsis famata }\end{array}$ & $\begin{array}{l}- \\
\bar{H} \\
H \\
H\end{array}$ & $\begin{array}{l}\text { H } \\
H \\
H \\
H \\
H\end{array}$ & $\begin{array}{l}\text { Ht } \\
\text { H } \\
\text { H } \\
\text { Ht } \\
\text { Ht }\end{array}$ & $\begin{array}{l}- \\
\overline{-} \\
-\end{array}$ & $\begin{array}{l}- \\
- \\
-\end{array}$ & $\begin{array}{l}- \\
- \\
- \\
-\end{array}$ \\
\hline $\begin{array}{l}\text { No. } 184 \\
\text { No. } 186 \\
\text { No. } 196 \\
\text { No. } 199 \\
\text { No. } 202\end{array}$ & & $\begin{array}{l}\text { Ht } \\
\text { H } \\
H+ \\
\text { H } \\
-\end{array}$ & $\begin{array}{l}\text { H } \\
H \\
H \\
H \\
H\end{array}$ & $\begin{array}{l}\text { Ht } \\
H \\
H \\
H \\
H \\
H\end{array}$ & $\begin{array}{l}- \\
\bar{H} \\
+ \\
+\end{array}$ & $\begin{array}{l}- \\
- \\
-\end{array}$ & $\begin{array}{l}- \\
- \\
-\end{array}$ \\
\hline $\begin{array}{lr}\text { No. } & 74 \\
\text { No. } & 75 \\
\text { No. } & 76 \\
\text { No. } 116 \\
\text { No. } 20\end{array}$ & $\begin{array}{l}\text { T. inconspicua } \\
\text { Trichosporon behrendii }\end{array}$ & $\begin{array}{l}- \\
E \\
E \\
z\end{array}$ & $\begin{array}{l}\text { H } \\
H \\
H \\
H \\
H \\
H\end{array}$ & $\begin{array}{l}\text { H } \\
\text { H } \\
\text { H } \\
\text { H } \\
H\end{array}$ & $\begin{array}{l}\text { Hit } \\
\text { Hit } \\
\text { HH } \\
\text { Hi } \\
\text { H }\end{array}$ & $\begin{array}{l}H \\
H \\
H \\
H \\
H\end{array}$ & $\begin{array}{l}- \\
- \\
-\end{array}$ \\
\hline $\begin{array}{lr}\text { No. } & 27 \\
\text { No. } & 129 \\
\text { No. } & 130 \\
\text { No. } 189 \\
\text { No. } 18\end{array}$ & Tr. infestans & $\begin{array}{l}H \\
H \\
H \\
H \\
+\end{array}$ & $\begin{array}{l}\text { H } \\
\text { H } \\
H \\
H \\
H\end{array}$ & $\begin{array}{l}H+H \\
H \\
H \\
H \\
H \\
H\end{array}$ & $\begin{array}{l}\overline{-} \\
\text { Hit } \\
\text { HH } \\
\text { Hit }\end{array}$ & $\underline{+}$ & $\begin{array}{l}- \\
\overline{-} \\
-\end{array}$ \\
\hline $\begin{array}{lr}\text { No. } 173 \\
\text { No. } \\
\text { No. } 152 \\
\text { No. } 144\end{array}$ & $\begin{array}{l}T r . \text { pullulans } \\
\text { Black yeast } \\
\text { yeast-like fungi }\end{array}$ & $\begin{array}{l}-\bar{H} \\
+ \\
+\end{array}$ & $\begin{array}{l}H+H \\
H \\
H \\
H\end{array}$ & $\frac{H H}{H+H}$ & $\begin{array}{l}- \\
\bar{H} \\
+\end{array}$ & $\begin{array}{l}- \\
- \\
-\end{array}$ & - \\
\hline
\end{tabular}


Table 3. The growth in peptone sea water.

\begin{tabular}{|c|c|c|c|c|c|}
\hline Code & Taxon & Growth & Code & Taxon & Growth \\
\hline $\begin{array}{lr}\text { No. } & 1 \\
\text { No. } & 14 \\
\text { No. } & 16 \\
\text { No. } & 10 \\
\text { No. } & 24\end{array}$ & $\begin{array}{l}\text { Saccharomyces exiguus } \\
\text { S. cerevisiae } \\
\text { S. delbrueckii } \\
\text { Hansenula anomala } \\
\text { Candida parapsilosis } \\
\quad \text { var. intermedia }\end{array}$ & $\begin{array}{l}- \\
- \\
+ \\
+\end{array}$ & $\begin{array}{l}\text { No. } 139 \\
\text { No. } 158 \\
\text { No. } 172 \\
\text { No. } 182 \\
\text { No. } 109\end{array}$ & C. sp. & $\begin{array}{l}\text { Ht } \\
+ \\
+ \\
+ \\
+\end{array}$ \\
\hline $\begin{array}{l}\text { No. } 164 \\
\text { No. } 165 \\
\text { No. } 169 \\
\text { No. } 170 \\
\text { No. } 177\end{array}$ & & $\begin{array}{l}H \\
H \\
+ \\
+ \\
-\end{array}$ & $\begin{array}{l}\text { No. } 110 \\
\text { No. } 122 \\
\text { No. } 146 \\
\text { No. } 147 \\
\text { No. } 148\end{array}$ & & $\begin{array}{l}+ \\
+ \\
+ \\
H \\
H\end{array}$ \\
\hline $\begin{array}{l}\text { No. } 181 \\
\text { No. } 185 \\
\text { No. } 187 \\
\text { No. } 192 \\
\text { No. } 193\end{array}$ & & $\frac{\bar{t}}{-}$ & $\begin{array}{l}\text { No. } 149 \\
\text { No. } 150 \\
\text { No. } 151 \\
\text { No. } 152 \\
\text { No. } 153\end{array}$ & & $\begin{array}{l}+ \\
+ \\
+ \\
+ \\
+\end{array}$ \\
\hline $\begin{array}{l}\text { No. } 194 \\
\text { No. } 197 \\
\text { No. } 200 \\
\text { No. } 203 \\
\text { No. } 204\end{array}$ & & $\frac{H}{-}$ & $\begin{array}{lr}\text { No. } & 154 \\
\text { No. } & 159 \\
\text { No. } & 160 \\
\text { No. } & 2 \\
\text { No. } & 99\end{array}$ & C. brumptii & $\begin{array}{l}\text { Ht } \\
+ \\
+ \\
+ \text { Ht }\end{array}$ \\
\hline $\begin{array}{lr}\text { No. } & 84 \\
\text { No. } & 98 \\
\text { No. } & 100 \\
\text { No. } 112 \\
\text { No. } 114\end{array}$ & & $\begin{array}{l}H \\
+ \\
- \\
-\end{array}$ & $\begin{array}{rr}\text { No. } & 117 \\
\text { No. } & 82 \\
\text { No. } & 17 \\
\text { No. } & 29 \\
\text { No. } & 34\end{array}$ & $\begin{array}{l}\text { C. solani } \\
\text { C. mycoderma } \\
\text { C. albicans } \\
\text { C. guilliermondii }\end{array}$ & $\frac{H}{-H}$ \\
\hline $\begin{array}{lr}\text { No. } & 115 \\
\text { No. } & 163 \\
\text { No. } & 167 \\
\text { No. } & 62 \\
\text { No. } & 63\end{array}$ & & $\begin{array}{l}- \\
+ \\
+ \\
+\end{array}$ & $\begin{array}{rr}\text { No. } & 7 \\
\text { No. } & 66 \\
\text { No. } & 67 \\
\text { No. } & 68 \\
\text { No. } & 36\end{array}$ & $\begin{array}{l}\text { C. intermedia } \\
\text { C. lipolytica } \\
\begin{array}{c}\text { Rhodotorula } \\
\text { mucilaginosa }\end{array}\end{array}$ & $\begin{array}{l}\text { Ht } \\
\text { Ht } \\
\text { Ht } \\
\text { Ht } \\
-\end{array}$ \\
\hline $\begin{array}{lr}\text { No. } & 69 \\
\text { No. } & 70 \\
\text { No. } & 71 \\
\text { No. } & 31 \\
\text { No. } 106\end{array}$ & C. tropicalis & $\begin{array}{l}+H \\
+ \\
+ \\
+\end{array}$ & $\begin{array}{ll}\text { No. } & 38 \\
\text { No. } & 61 \\
\text { No. } & 78 \\
\text { No. } & 80 \\
\text { No. } & 89\end{array}$ & & $\frac{-}{+}$ \\
\hline $\begin{array}{l}\text { No. } 123 \\
\text { No. } 124 \\
\text { No. } 131 \\
\text { No. } 132 \\
\text { No. } 135\end{array}$ & & $\begin{array}{l}H \\
H \\
+ \\
H \\
H\end{array}$ & $\begin{array}{l}\text { No. } 127 \\
\text { No. } 128 \\
\text { No. } 136 \\
\text { No. } 161 \\
\text { No. } 171\end{array}$ & & $\begin{array}{l}+ \\
H+1 \\
H+ \\
H \\
H\end{array}$ \\
\hline
\end{tabular}


Table 3. Continued.

\begin{tabular}{|c|c|c|c|c|c|}
\hline Code & Taxon & Growth & Code & Taxon & Growth \\
\hline $\begin{array}{l}\text { No. } 174 \\
\text { No. } 179 \\
\text { No. } 188 \\
\text { No. } 190 \\
\text { No. } 195\end{array}$ & & $\begin{array}{l}\text { Ht } \\
+H \\
+H \\
+H \\
+\end{array}$ & $\begin{array}{l}\text { No. } 186 \\
\text { No. } 196 \\
\text { No. } 199 \\
\text { No. } 202 \\
\text { No. } 74\end{array}$ & T. inconspicua & $\frac{t}{ \pm}$ \\
\hline $\begin{array}{lr}\text { No. } 201 \\
\text { No. } 104 \\
\text { No. } 88 \\
\text { No. } 94 \\
\text { No. } 95\end{array}$ & $\begin{array}{l}\text { Rh. minuta } \\
\text { Rh. flava }\end{array}$ & $\begin{array}{l}\overline{+} \\
\frac{5}{+}\end{array}$ & 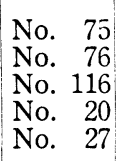 & $\begin{array}{l}\text { Trichosporon behrendii } \\
\text { Tr. cutancum }\end{array}$ & $\frac{-}{+}$ \\
\hline $\begin{array}{l}\text { No. } 77 \\
\text { No. } 85 \\
\text { No. } 162 \\
\text { No. } 35 \\
\text { No. } 64\end{array}$ & $\begin{array}{l}\text { Rh. glutinis } \\
\text { Cryptococcus laurentii }\end{array}$ & $\frac{ \pm}{t}$ & \begin{tabular}{|l|} 
No. 129 \\
No. 130 \\
No. 189 \\
No. 18 \\
No. 173
\end{tabular} & Tr. infestans & $\begin{array}{l}H \\
H \\
H \\
H \\
H \\
H\end{array}$ \\
\hline $\begin{array}{ll}\text { No. } & 65 \\
\text { No. } & 72 \\
\text { No. } & 79 \\
\text { No. } & 83 \\
\text { No. } & 92\end{array}$ & & $\begin{array}{l}- \\
\pm \\
\pm\end{array}$ & $\mid \begin{array}{l}\text { No. } 22 \\
\text { No. } 155 \\
\text { No. } 144\end{array}$ & $\begin{array}{l}\text { Tr. pullulans } \\
\text { Black yeast } \\
\text { yeast-like fungi }\end{array}$ & $\begin{array}{l}+ \\
+ \\
+t\end{array}$ \\
\hline $\begin{array}{l}\text { No. } 111 \\
\text { No. } 143 \\
\text { No. } 176 \\
\text { No. } 178 \\
\text { No. } 108\end{array}$ & Cr. albidus & $\begin{array}{l}+ \\
+ \\
+\end{array}$ & & & \\
\hline $\begin{array}{l}\text { No. } 119 \\
\text { No. } 120 \\
\text { No. } 125 \\
\text { No. } 133 \\
\text { No. } 183\end{array}$ & & $\begin{array}{l}- \\
\bar{E} \\
+\end{array}$ & & & \\
\hline $\begin{array}{l}\text { No. } 86 \\
\text { No. } 175 \\
\text { No. } 93 \\
\text { No. } 191 \\
\text { No. } 101\end{array}$ & $\begin{array}{l}\text { Cr. diffuens } \\
\text { Cr. neoformans } \\
\text { Cr. luteolus }\end{array}$ & $\begin{array}{l}+ \\
+ \\
+ \\
+\end{array}$ & & & \\
\hline $\begin{array}{l}\text { No. } 156 \\
\text { No. } \\
\text { No. } 168 \\
\text { No. } 180 \\
\text { No. } 184\end{array}$ & Torulopsis famata & $\begin{array}{l}+ \\
+ \\
+ \\
+ \\
+ \\
+\end{array}$ & & & \\
\hline
\end{tabular}

